\title{
Irritative and sensory disturbances in oral implantology. Literature review
}

\author{
Cristina Palma-Carrió ${ }^{1}$, Jose Balaguer-Martínez ${ }^{2}$, David Peñarrocha-Oltra ${ }^{1}$, MaríaPeñarrocha-Diago ${ }^{2}$ \\ ${ }^{1}$ Resident, Master of Oral Surgery and Implantology. Valencia University Medical and Dental School, Spain \\ ${ }^{2}$ Associate Professor, Master of Oral Surgery and Implantology. Valencia University Medical and Dental School, Spain
}

Correspondence:

Clínicas Odontológicas,

Gascó Oliag, 1,

46021 Valencia (Spain)

maria.penarrocha@uv.es

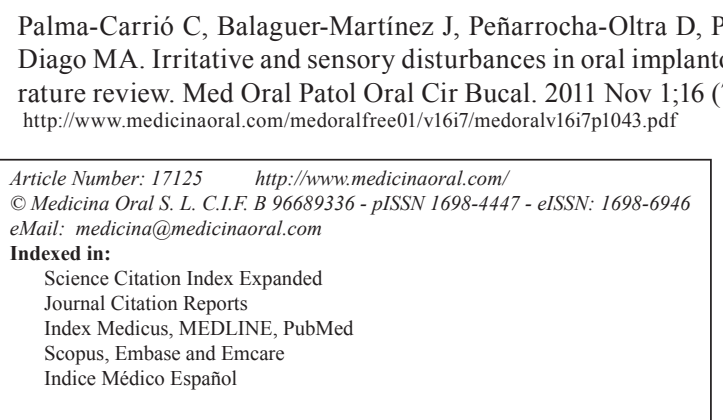

\begin{abstract}
The aim of this study was to review irritative and sensory disturbances following placement of dental implants. A literature search was made of PubMed for articles published between 2000 and 2010. Studies that reported sensory disturbances directly caused by the placement of dental implants were included.

Sensory deficits or trigeminal neuropathy are caused by damage to the third branch of the trigeminal nerve during surgery. This manifests in the immediate postoperative period as a sensory deficit not usually associated with pain and generally transient. The literature reviewed reported irritative and sensory disturbances caused during surgery, after surgery, and as a result of complications. Postoperative pain appears after oral surgery as a result of inflammation associated with damage to tissue during surgery. Pain due to postoperative complications following implant placement was classified as neurogenic pain, peri-implant pain and bone pain.
\end{abstract}

Key words: Neurosensory disturbance, pain, dental implants, postoperative complications.

\section{Introduction}

Sensory deficits of the trigeminal nerve manifest as numbness, sometimes associated with dysesthesia or paresthesia, described as an unpleasant sensation, and rarely painful (1), being dental treatment the most common cause (2). The most frequently injured trigeminal nerve branches in oral surgery are the inferior alveolar nerve (IAN), the mental nerve and the lingual nerve. These lesions have many causes such as surgical sectioning of the third molar, removal of bone, surgical incision, postoperative edema, hematoma formation, postoperative infection, implant surgery and injection of local anesthetic (3).

Irritative sensory disturbances in oral implantology may occur during surgery, during the postoperative period and after complications. Most patients do not report pain during implant surgery, where pain is related to the number of implants placed (4) and anxiety (5). Several articles have studied pain and swelling secondary to procedures such as removal of impacted third molars (6), periapical surgery (7) and implant placement $(4,8)$. A review by Greenstein et al. (9) reported clinical 
recommendations for preventing and managing surgical complications associated with implant placement; complications causing pain were nerve injury, wound dehiscence, injury to an adjacent tooth, mandibular fracture and maxillary sinusitis. Cases of osteomyelitis (10) and persistent idiopathic facial pain have been reported (11).

The aim of this study was to review the literature on sensory disturbances caused by the placement of dental implants.

\section{Material and Methods}

We conducted a PubMed search for articles published between 2000 and 2010 that evaluated complications after implant placement. The keywords used were 'neurosensory disturbances', 'pain', 'dental implants' and 'postoperative complications'. Studies published in English or Spanish dental journals were included. Animal studies and those for which the full text could not be obtained were excluded. We included 24 studies that reported irritative or sensory disturbances occurring directly after implant placement.

\section{Results}

Sensory deficits

Occasionally during implant surgery, nerve damage occurs which produces neurosensory consequences in the immediate postoperative period. Nerve injury is most frequently caused by extraction of a mandibular third molar $(12,13)$, and is considered one of the possible complications of implant placement along with transposition of the IAN (14). Tay and Zuniga (13), studied the etiology and characteristics of 73 lesions of the trigeminal nerve in 59 patients; the most frequent cause was mandibular third molar surgery, followed by injection of local anesthetics, implant surgery and orthognathic surgery; the most affected trigeminal nerve branch was the inferior alveolar nerve (IAN) followed by the lingual nerve; implant surgery was responsible for $14.9 \%$ of the injuries to the IAN. Bartling et al. (15) evaluated the incidence of altered sensation to the mental nerve following placement of 405 mandibular implants in 94 patients; 8 patients $(8.5 \%)$ had transient altered sensation without presenting hyperesthesia or dysesthesia.

Before implant placement, the clinician should check for the presence of sensory disturbances. When implants are placed close to the mental foramen, the anterior loop of the nerve should be taken into consideration (16). The panoramic radiograph is a safe preoperative diagnostic method for posterior mandibular implants (17), the location of implants $2 \mathrm{~mm}$ above the inferior alveolar canal based on panoramic images, and $1 \mathrm{~mm}$ above the canal based on computed tomography is recommended (15).

Recovery of sensation is faster during the first 6 months after nerve injury and is not related to age or sex; howe- ver, recovery is related to the etiology of the lesion, surgical removal of a third molar being more easily recovered from than after other causes such as endodontic treatments, implant surgery and injection of anesthetic (12). The early removal of implants associated with IAN injury (before 36 hours postoperative) minimizes or resolves IAN neuropathy. Adjunctive therapy of high dose corticosteroids and nonsteroidal antiinflammatory drugs can also help (18).

Irritative sensory disturbances Intraoperative pain

Al-Khabbaz et al. (4) studied pain during the placement of 510 implants in 234 patients; $84.2 \%$ of patients had no pain, $12.8 \%$ had mild pain and $2.6 \%$ moderate pain; only one patient had severe pain. Pain was higher in patients who received multiple implants, and in implants placed by periodontists versus those placed by graduate students; although this may be because the number of implants per patient was higher for periodontists than for students. Pain during surgery was an indicator of pain at 24 hours, and pain at 24 hours was an indicator of pain at one week.

Postoperative pain (side effects)

The correct management of patient anxiety can play an important role in reducing the subjective experience of pain (5). After dental implant surgery, patients present varying degrees of pain and swelling as a direct result of surgery, which are related to the number of implants placed (8). In a series 41 patients (8) 131 dental implants were placed, observing peak pain at 6 hours after surgery in $41.5 \%$ of patients. Al-Khabbaz et al. (4) observed that after 24 hours, $80.3 \%$ of patients had some pain, the majority of which was mild (69.7\%); after one week $60.3 \%$ of patients still had some pain, whereas at 6 weeks $5.1 \%$ of patients had mild pain, and none of the patients had pain at 12 weeks. At 24 hours, pain was significantly related to the implants placed in women, implants placed by graduate students, and with the presence of pain during surgery. A randomized controlled study (19), showed that flapless implant placement caused less pain for less time than with open-flap surgery. Similar results were obtained by Cannizzaro et al. (20), in which the flap elevation group had significantly more postoperative pain and edema, and increased consumption of analgesics.

\section{Neurogenic pain}

Neurogenic pain may be associated with nerve injury (13). If this occurs during administration of local anesthetics or during implant surgery, the patient sometimes experiences the sensation of electric shock or severe pain. Hillerup (12) studied 52 patients with IAN lesions from iatrogenic causes (36 for third molar surgery, 5 for local anesthetic injection, 5 for implant surgery, 4 for endodontic treatment and 2 for unknown reasons), only 5 patients (10\%) had no neurogenic symptoms, while 
the majority of patients (29-56\%) had paresthesia, and 9 (17\%) had permanent or recurrent dysesthesia. Tay and Zuniga (13), evaluated 73 lesions of the trigeminal nerve in 59 patients; the neuropathic pain was present in 7 cases (14.9\% of the IAN lesions) with mild or no sensory deficit, neuropathic pain being more probable in minor nerve lesions. Leckel et al. (21) described a rare case of persistent neuropathic pain without hyperesthesia or dysesthesia after the placement of 2 dental implants in the posterior mandibular region; computed tomography revealed the close proximity of the apex of the implant to the mandibular canal; following removal of the dental implants the patient had no pain at one year.

\section{Peri-implant pain}

Infection around the apical portion of the implant is known as apical periimplantitis (22); it can be caused by contamination at the time of instrumentation, by overheating of bone, or the presence of a pre-existing bone disease. Periapical implant disease is classified according to the evolutive stage as either acute lesions (nonsuppurative and suppurative) or as chronic (or periapical abscesses) (23).

Clinically, in the acute implant periapical lesion (nonsuppurative and suppurative) spontaneous acute pain appears in the area of the affected implant, which does not increase on percussion of the implant due to the direct bone-implant interface; as well as gingival inflammation in the area adjacent to the periapex. Radiologically, there are no changes in a non-suppurative lesion, while in the suppurative lesion a periapical radiolucency appears. Treatment of acute suppurative and non-suppurative apical periimplantitis is periapical surgery with curettage and irrigation (23), although curettage with resection of the apical portion of the affected implant has also been described (24). Peñarrocha et al. (25) evaluated 7 cases with acute apical periimplantitis 7 to 15 days after implant placement, all patients reported pain in the periapical area of the implant and were treated with periapical surgery, the pain and swelling decreased one week after treatment.

In chronic lesions or periapical abscesses, symptoms are rare except in the exacerbation phase, implant mobility is present, as well as suppuration and gingival reddening. Radiologically, implant periapical radiolucency with destruction of marginal bone is present; treatment is removal of the implant (23).

\section{Bone pain}

Bisphosphonates (BFs) are a group of drugs that inhibit bone resorption. Intravenous BFs are often used to reduce bone pain in malignant hypercalcemia, Paget's disease and bone metastases (26). Oral BFs are used to treat osteoporosis, Paget's disease and osteogenesis imperfecta. Osteonecrosis of the jaw following oral surgery, including implant placement, has been described in patients taking BFs (27), and tooth extractions are the main cause (28-30). Few articles in the reviewed literature $(26,31,32)$ report cases of osteonecrosis associated with BFs after implant placement which may be due to the low frequency of implant placement in patients undergoing BF treatment (32). Lazorovici et al. (32) recently presented the largest series of osteonecrosis of the jaw caused by BF associated with dental implants. Of the 145 patients diagnosed with osteonecrosis, 27 (18.6\%) were associated with implant placement, 6 patients developed osteonecrosis during the first 6 months after implant placement.

For the diagnosis of osteonecrosis, 3 features are present: 1) current or previous treatment with $\mathrm{BF}, 2$ ) exposure of bone in the maxillofacial region for more than 8 weeks and 3) no history of radiation treatment of the jaws. Treatment of osteonecrosis depends on the stage at which the disease is found. In stage 0 , there is no bone exposure and the symptoms and clinical findings are nonspecific; treatment is systemic pain medication and antibiotics. In stage 1, asymptomatic bone exposure occurs without apparent infection; treatment consists of antibacterial mouthwash. Stage 2 is characterized by bone exposure and bone necrosis in patients with pain and clinical evidence of infection with or without purulent drainage; treatment is symptomatic with oral antibiotics, with antibacterial mouthwash, pain management and superficial debridement. In stage 3, bone exposure and bone necrosis occur with pain and infection, presenting one or more of the following conditions: exposure of necrotic bone beyond the alveolar bone region, pathologic fracture, extraoral fistula, oral-antral communication and osteolysis. Treatment consists of antibacterial mouthwash, antibiotic therapy, pain management and surgical debridement (33).

\section{Conclusions}

Sensory alterations of the trigeminal nerve after implant placement are rare and usually affect the inferior alveolar nerve. The most common irritative sensory disturbance following implant placement is postoperative pain, the intensity of which is related to the number of implants placed. Pain following postoperative complications is poorly documented and may appear as a result of trigeminal nerve injury, mandibular fracture, apical periimplantitis, and osteonecrosis caused by bisphosphonates.

\section{References}

References with links to Crossref - DOI

1. Peñarrocha M, Cervelló MA, Martí E, Bagán JV. Trigeminal neuropathy. Oral Dis. 2007;13:141-50.

2. Kalladka M, Proter N, Benoliel R, Czerninski R, Eliav E. Mental nerve neuropathy: patient characteristics and neurosensory changes. Oral Surg Oral Med Oral Pathol Oral Radiol Endod. 2008;106:36470 
3. Carnevali R, De Filippo C. [Lesions of the inferior alveolar nerve]. Minerva Stomatol. 1989;38:1015-9.

4. Al-Khabbaz AK, Griffin TJ, Al-Shammari KF. Assessment of pain associated with the surgical placement of dental implants. J Periodontol. 2007;78:239-46.

5. Hashem AA, Claffey NM, O'Connell B. Pain and anxiety following the placement of dental implants. Int J Oral Maxillofac Implants. 2006;21:943-50.

6. Olmedo-Gaya MV, Vallecillo-Capilla M, Galvez-Mateos R. Relation of patient and surgical variables to postoperative pain and inflammation in the extraction of third molars. Med Oral. 2002;7:360-9.

7. García B, Larrazabal C, Peñarrocha M, Peñarrocha M. Pain and swelling in periapical surgery. A literature update. Med Oral Patol Oral Cir Bucal. 2008;13:E726-9.

8. González-Santana H, Peñarrocha-Diago M, Guarinos-Carbó J, Balaguer-Martínez J. Pain and inflammation in 41 patients following the placement of 131 dental implants. Med Oral Patol Oral Cir Bucal. 2005;10:258-63.

9. Greenstein G, Cavallaro J, Romanos G, Tarnow D. Clinical recommendations for avoiding and managing surgical complications associated with implant dentistry: a review. J Periodontol. 2008;79:131729.

10. Rokadiya S, Malden NJ. An implant periapical lesion leading to acute osteomyelitis with isolation of Staphylococcus aureus. Br Dent J. 2008;205:489-91.

11. Queral-Godoy E, Vazquez-Delgado E, Okeson JP, Gay-Escoda C. Persistent idiopathic facial pain following dental implant placement: a case report. Int J Oral Maxillofac Implants. 2006;21:136-40.

12. Hillerup S. Iatrogenic injury to the inferior alveolar nerve: etiology, signs and symptoms, and observations on recovery. Int J Oral Maxillofac Surg. 2008;37:704-9.

13. Tay AB, Zuniga JR. Clinical characteristics of trigeminal nerve injury referrals to a university centre. Int J Oral Maxillofac Surg. 2007;36:922-7.

14. Luna AH, Passeri LA, de Moraes M, Moreira RW. Endosseous implant placement in conjunction with inferior alveolar nerve transposition: a report of an unusual complication and surgical management. Int J Oral Maxillofac Implants. 2008;23:133-6.

15. Bartling R, Freeman K, Kraut RA. The incidence of altered sensation of the mental nerve after mandibular implant placement. J Oral Maxillofac Surg. 1999;57:1408-12.

16. Kraut RA, Chahal O. Management of patients with trigeminal nerve injuries after mandibular implant placement. J Am Dent Assoc. 2002;133:1351-4.

17. Vazquez L, Saulacic N, Belser U, Bernard JP. Efficacy of panoramic radiographs in the preoperative planning of posterior mandibular implants: a prospective clinical study of 1527 consecutively treated patients. Clin Oral Implants Res. 2008;19:81-5.

18. Khawaja N, Renton T. Case studies on implant removal influencing the resolution of inferior alveolar nerve injury. Br Dent $\mathrm{J}$. 2009;206:365-70.

19. Fortin T, Bosson JL, Isidori M, Blanchet E. Effect of flapless surgery on pain experienced in implant placement using an imageguided system. Int J Oral Maxillofac Implants. 2006;21:298-304.

20. Cannizzaro G, Leone M, Consolo U, Ferri V, Esposito M. Immediate functional loading of implants placed with flapless surgery versus conventional implants in partially edentulous patients: a 3-year randomized controlled clinical trial. Int J Oral Maxillofac Implants. 2008;23:867-75.

21. Leckel M, Kress B, Schmitter M. Neuropathic pain resulting from implant placement: case report and diagnostic conclusions. J Oral Rehabil. 2009;36:543-6.

22. Quirynen M, Gijbels F, Jacobs R. An infected jawbone site compromising successful osseointegration. Periodontol 2000. 2003;33:129-44.

23. Peñarrocha Diago M, Boronat López A, Lamas Pelayo J. Update in dental implant periapical surgery. Med Oral Patol Oral Cir Bucal. 2006;11:E429-32.
24. Dahlin C, Nikfarid H, Alsén B, Kashani H. Apical peri-implantitis: possible predisposing factors, case reports, and surgical treatment suggestions. Clin Implant Dent Relat Res. 2009;11:222-7.

25. Peñarrocha-Diago M, Boronat-Lopez A, García-Mira B. Inflammatory implant periapical lesion: etiology, diagnosis, and treatment-presentation of 7 cases. J Oral Maxillofac Surg. 2009;67:168-73.

26. Marx RE, Sawatari Y, Fortin M, Broumand V. Bisphosphonateinduced exposed bone (osteonecrosis/osteopetrosis) of the jaws: risk factors, recognition, prevention, and treatment. J Oral Maxillofac Surg. 2005;63:1567-75.

27. Flichy-Fernández AJ, Balaguer-Martínez J, Peñarrocha-Diago M, Bagán JV. Bisphosphonates and dental implants: current problems. Med Oral Patol Oral Cir Bucal. 2009;14:E355-60.

28. Bagan JV, Murillo J, Jimenez Y, Poveda R, Milian MA, Sanchis $\mathrm{JM}$, et al. Avascular jaw osteonecrosis in association with cancer chemotherapy: series of 10 cases. J Oral Pathol Med. 2005;34:120-3. 29. Bagan JV, Jimenez Y, Murillo J, Hernandez S, Poveda R, Sanchis JM, et al. Jaw osteonecrosis associated with bisphosphonates: multiple exposed areas and its relationship to teeth extractions. Study of 20 cases. Oral Oncol. 2006;42:327-9.

30. Montoya-Carralero JM, Parra-Mino P, Ramírez-Fernández P, Morata-Murcia IM, Mompeán-Gambín Mdel C, Calvo-Guirado JL. Dental implants in patients treated with oral bisphosphonates: a bibliographic review. Med Oral Patol Oral Cir Bucal. 2010;15:e65-9.

31. Marx RE, Cillo JE Jr, Ulloa JJ. Oral bisphosphonate-induced osteonecrosis: risk factors, prediction of risk using serum CTX testing, prevention, and treatment. J Oral Maxillofac Surg. 2007;65:2397410.

32. Lazarovici TS, Yahalom R, Taicher S, Schwartz-Arad D, Peleg $\mathrm{O}$, Yarom N. Bisphosphonate-related osteonecrosis of the jaw associated with dental implants. J Oral Maxillofac Surg. 2010;68:790-6. 33. Colella G, Campisi G, Fusco V. American Association of Oral and Maxillofacial Surgeons position paper: Bisphosphonate-Related Osteonecrosis of the Jaws-2009 update: the need to refine the BRONJ definition. J Oral Maxillofac Surg. 2009;67:2698-9. 